\title{
Biology Dictates Prognosis Following Resection of Gallbladder Carcinoma: Sometimes Less is More
}

\author{
Timothy M. Pawlik, MD, MPH and Michael A. Choti, MD, MBA \\ Division of Surgical Oncology, Sidney Kimmel Comprehensive Cancer Center at Johns Hopkins, Baltimore, MD
}

In this issue of Annals of Surgical Oncology, D’Angelica and colleagues from the Memorial Sloan-Kettering Cancer Center report data on the importance of extent of resection for gallbladder adenocarcinoma. The analyses reveal that extensive resections for gallbladder cancer are associated with increased morbidity, but not survival. These findings have important implications as some investigators have in the past advocated extended operations that combine major hepatic resection and routine common bile duct resection with reconstructive hepaticojejunostomy to improve longterm survival. ${ }^{1,2}$ Prognosis following surgery for gallbladder adenocarcinoma can vary dramatically, with 5-year survival rates ranging from $10 \%$ to $90 \%$. As noted by the authors in their current report, outcome following surgery depends not only on the extent of the curative resection, but more importantly, on the stage of disease. ${ }^{3}$ D'Angelica and colleagues reaffirm the basic oncologic tenet that tumor biology-not extent of resection-is the dominate factor that dictates survival.

Whether to perform empirically a nonanatomical resection or an anatomical resection for hepatobiliary malignancies of the liver has been controversial. ${ }^{4,5}$ Although some studies have reported that the performance of an anatomical resection improves tumor clearance and outcome, other reports have not demonstrated a benefit for anatomic or "extended" resections. ${ }^{4,6}$ In general, most surgeons have begun to advocate a more parenchymalsparing approach to resection of hepatobiliary tumors that still maintains a negative margin. In support of this, our group previously reported that the extent of hepatectomy for gallbladder carcinoma was not associated with prognosis. ${ }^{6}$ D'Angelica similarly noted that patients who

(c) Society of Surgical Oncology 2009

Published Online: 23 January 2009

M. A. Choti, MD, MBA

e-mail: mchoti@jhmi.edu underwent a major hepatectomy had a 5-year actuarial survival comparable to those patients who did not undergo major hepatic resection. However, unlike D' Angelica et al., we previously reported that, rather than extent of hepatic resection, surgical margin status was a key determinant of outcome. Specifically, R1/R2 margin status was associated with significantly worse long-term outcome. ${ }^{6}$ The failure of the current authors to find margin status to be a significant prognostic factor may be due to the laudatory fact that only nine patients in the series had a positive resection margin. As such, analyses involving margin status may have been underpowered. Regardless, we agree whole-heartedly with our colleagues that rather than dogmatically adhering to an extended versus nonextended surgical resection approach, the surgeon's goal should be to resect all disease with negative histologic margins.

Similar to the extent of hepatic resection, the wholesale adoption of a more extensive approach to the common bile duct may also be unwarranted. While some surgeons have advocated routine resection of the common bile duct at the time of curative resection and portal lymphadenectomy, other investigators have questioned this approach. ${ }^{1,6,7}$ The study by D'Angelica et al., as well as others, now provide data to show that routine bile duct excision is not necessarily associated with improved overall survival. ${ }^{6,8}$ The notion that resection of the common bile duct may facilitate a more thorough lymphadenectomy also has been questioned. As noted in the D'Angelica report, lymph node counts were similar regardless of whether a common bile duct resection had been performed. It is important to note that, while routine common bile duct resection was not associated with survival or lymph node yield, it was associated with increased morbidity. In fact, D'Angelica et al. noted a $33 \%$ incidence of grade $3 / 4$ morbidity in the group of patients who underwent bile duct excision and reconstruction. Citing similar data showing no improvement in long-term survival, as well as the increased risk of 
complications following a bilioenteric anastomosis, the Makuuchi group has similarly recommended preservation of the extrahepatic bile duct when possible. ${ }^{8,9}$ Rather than adopting a universal approach to management of the common bile duct, a more selective approach may be warranted. Noting that a positive cystic duct marginbased either on the initial cholecystectomy specimen or intraoperative biopsy of the cystic duct stump-is strongly associated with residual disease in the common bile duct, our group has advocated selective common bile duct resection in conjunction with lymphadenectomy in this subset of patients in order to obtain an R0 resection. ${ }^{6}$

Similar to previous studies, D' Angelica et al. identified a number of prognostic factors that stratified patients with regard to prognosis following resection for gallbladder carcinoma. ${ }^{10,11}$ Specifically, factors associated with poor prognosis included advanced $\mathrm{T}$ - and American Joint Committee on Cancer (AJCC) stage, as well as metastatic nodal disease. Some surgeons have suggested that lymph node metastasis should be a relative contraindication to resection because poor survival is the rule. ${ }^{12}$ It should be noted, however, that in the current study the overall 5-year survival for patients with lymph node metastasis was about $20 \%$. As such, resection of patients with lymph node metastasis should still be considered. These data do, however, serve to emphasize the importance of tumor biology in ultimately dictating prognosis. Much more than operative technique, tumor biology is the ultimate adjudicator in determining patient long-term survival.

In summary, the authors are to be congratulated for an insightful analysis of such a large single-institution series of patients with gallbladder adenocarcinoma. Data from the current study emphasized that extent of hepatic resection does not appear to affect outcome. It is critical, however, to perform the appropriate operation necessary to achieve negative surgical margins. Sadly, gallbladder adenocarcinoma frequently manifests with aggressive tumor biology. It is unlikely that clinically significant improvements in survival will therefore result from more extensive resection of non-tumor-bearing hepatic parenchyma. In fact, such resections appear to be associated with increased morbidity. In light of these data from D'Angelica et al., for the surgical patient with gallbladder cancer sometimes less truly is more. Specifically, rather than a routine extended resection, a more rational approach to the patient with gallbladder cancer is to consider the extent and location of the tumor and to plan the extent of surgery based on what is necessary to achieve microscopically negative margins. Given the importance of tumor biology, future resources for investigation of gallbladder adenocarcinoma should be directed toward the study of emerging techniques for early diagnosis, as well as the development of novel, more efficacious systemic treatment strategies.

\section{REFERENCES}

1. Tsukada K, Hatakeyama K, Kurosaki I, Uchida K, Shirai Y, Muto T, et al. Outcome of radical surgery for carcinoma of the gallbladder according to the TNM stage. Surgery. 1996;120(5): 816-21.

2. Bartlett DL, Fong Y, Fortner JG, Brennan MF, Blumgart LH. Long-term results after resection for gallbladder cancer. Implications for staging and management. Ann Surg. 1996;224(5): 639-46.

3. Henson DE, Albores-Saavedra J, Corle D. Carcinoma of the gallbladder. Histologic types, stage of disease, grade, and survival rates. Cancer. 1992;70(6):1493-7.

4. Yasui K, Shimizu Y. Surgical treatment for metastatic malignancies. Anatomical resection of liver metastasis: indications and outcomes. Int J Clin Oncol. 2005;10(2):86-96.

5. Yamamoto J, Saiura A, Koga R, Seki M, Ueno M, Oya M, et al. Surgical treatment for metastatic malignancies. Nonanatomical resection of liver metastasis: indications and outcomes. Int J Clin Oncol. 2005;10(2):97-102.

6. Pawlik TM, Gleisner AL, Vigano L, Kooby DA, Bauer TW, Frilling A, et al. Incidence of finding residual disease for incidental gallbladder carcinoma: implications for re-resection. J Gastrointest Surg. 2007;11(11):1478-86; discussion 86-7.

7. Nakamura S, Sakaguchi S, Suzuki S, Muro H. Aggressive surgery for carcinoma of the gallbladder. Surgery. 1989;106(3):467-73.

8. Chijiiwa K, Tanaka M. Indications for and limitations of extended cholecystectomy in the treatment of carcinoma of the gall bladder. Eur J Surg. 1996;162(3):211-6.

9. Kosuge T, Sano K, Shimada K, Yamamoto J, Yamasaki S, Makuuchi M. Should the bile duct be preserved or removed in radical surgery for gallbladder cancer? Hepatogastroenterology. 1999;46(28):2133-7.

10. Muratore A, Polastri R, Bouzari H, Vergara V, Capussotti L. Radical surgery for gallbladder cancer: a worthwhile operation? Eur J Surg Oncol. 2000;26(2):160-3.

11. Foster JM, Hoshi H, Gibbs JF, Iyer R, Javle M, Chu Q, et al. Gallbladder cancer: defining the indications for primary radical resection and radical re-resection. Ann Surg Oncol. 2007;14(2): 833-40.

12. Benoist S, Panis Y, Fagniez PL. Long-term results after curative resection for carcinoma of the gallbladder French University Association for Surgical Research. Am J Surg. 1998;175(2): 118-22. 